\title{
Efeito de um treinamento combinado de força e endurance sobre componentes corporais de mulheres na fase de perimenopausa
}

\author{
Mateus Rossato 1 \\ Maria A. Binotto 1 \\ Maria A. Roth ${ }^{2}$ \\ Haury Temp ${ }^{2}$ \\ Felipe P. Carpes ${ }^{3}$ \\ Jose L. Alonso 4 \\ Airton J. Rombaldi 5
}

https://doi.org/10.5628/rpcd.07.01.92

\author{
1 Laboratório de Biomecânica,C entro de Desportos da \\ Universidade Federal de Santa Catarina \\ Brasil Florianópolis, SC \\ 2 Faculdade M etodista, Faculdade de Educação Física \\ Santa Maria, RS \\ 3 Laboratório de Biomecânica, Centro de Educação Física e \\ D esportos da Universidade Federal de Santa M aria \\ Brasil Santa M aria, RS \\ 4 D epartamento de Ciências M orfofuncionales \\ Facultad De M edicina, U niversidad D e Cordoba, Espanha \\ ${ }^{5}$ Escola Superior de Educação Física \\ Universidade Federal de Pelotas, Brasil Pelotas, RS
}

\section{RESUMO}

O objectivo deste estudo foi verificar o efeito de um treinamento de força e endurance sobre componentes corporais de mulheres. Oito mulheres na fase de perimenopausa (massa corporal de $58,9 \pm 9,3 \mathrm{~kg}$, altura de $159 \pm 7 \mathrm{~cm}$ e idade de $48,6 \pm 2,1$ anos) participaram do estudo. A massa óssea (MO), massa gorda (MG) e a massa magra (MM) foram analisadas com um exame DEXA (D ual Energy X-Ray A bsorptiometry). O consumo máximo de oxigénio $\left(\mathrm{VO}_{2}\right.$ máx) foi determinado através de teste progressivo máximo em esteira rolante antes e após o treinamento. $\mathrm{O}$ treinamento combinando exercícios de força e endurance teve a duração de 20 semanas. Os resultados indicam que o $\mathrm{VO}_{2}$ máx, embora não alterado de maneira estatisticamente significativa, apresentou um aumento de $7,23 \%$ para a forma absoluta e $9,82 \%$ na forma relativa. Da mesma forma, a MO e MM tiveram um aumento de $0,81 \%$ e $2,82 \%$, respectivamente, enquanto que a MG apresentou decréscimo de 3,60\%. Ainda que essas alterações não tenham alcançado significância estatística, a tendência de modificação observada sugere que o treinamento poderá induzir alterações nos componentes corporais de mulheres na fase de perimenopausa. Para a confirmação dos resultados encontrados sugerem-se estudos com períodos de treinamento mais extensos.

Palavras-chave: mulheres; perimenopausa; consumo de oxigénio, treinamento.

\section{ABSTRACT}

Effects of a combined training of strength and endurance on body components of women on perimenopause stage

The purpose of this study was to verify the effects of strength and endurance training on body components in women. Eight women at perimenopause stage (age of $48.6 \pm 2.1$ years; body mass of $58.9 \pm 9.3 \mathrm{~kg}$; height of $159 \pm 7 \mathrm{~cm}$ ) volunteered for this study. The bone mass (BM), fat mass (FM) and lean mass (LM) were analyzed by a DEXA (Dual Energy X-Ray A bsorptiometry), and the $\mathrm{VO}_{2}$ max was determined by progressive maximal tests in a treadmill before and after the training period. The strength and endurance training had duration of 20 weeks. We didn't find statistical differences for the $\mathrm{VO}_{2} \max$, however presented an increase of $7.23 \%$ for absolute and 9.82 for relative values. The BM had an increase of $0.81 \%$, while the LM had an increase of $2.82 \%$. The FM was decreased in $3.60 \%$. Although the alterations did not reach a statistical significance, the tendency observed suggest that training can induce changes on the body components of women at perimenopause stage. However, to confirm this found is necessary the application of a larger training period.

Keys-words: women, perimenopause, oxygen uptake, training 


\section{INTRODUÇÃO}

O processo de envelhecimento é o principal factor responsável pelas modificações na composição corporal (7). No corpo feminino tais modificações se fazem mais visíveis, e têm sido objecto de estudos de muitos pesquisadores $(33,34)$.

Dentre as fases de envelhecimento aquela que antecede a menopausa é denominada como perimenopausa. Durante a perimenopausa, no que se refere à mudança dos componentes corporais, observa-se um aumento do percentual de massa gorda, passando dos 20 a $25 \%$ característicos da fase adulta jovem, para cerca de 30 a $35 \%(42,32)$, além de ser observada uma aceleração no processo natural de perda da massa óssea. Este fato acaba por contribuir para o surgimento de doenças como a osteoporose ${ }^{(19)}$, que se caracteriza pela fragilidade do tecido ósseo em relação a sobrecargas mecânicas, fazendo com o osso seja mais susceptível a fracturas $(21,1)$.

Svendsen et al. (36) afirmam que na fase de perimenopausa a diminuição na densidade mineral óssea é maior (alcançando o índice de 1,85\% ao ano, aproximadamente), especialmente quando se analisa a região da coluna lombar, quando comparada à fase da menopausa ( $1,29 \%$ ao ano). Além disso, mulheres na fase de perimenopausa apresentam um decréscimo gradativo da massa óssea e massa corporal total, bem como um aumento da gordura corporal e da gordura visceral, favorecendo com isso, o aumento de factores de riscos cardiovasculares e de doenças de cunho metabólico, como a diabetes e dislipidemia (20).

Para combater essas perdas a prática de exercício físico regular tem sido um dos principais métodos aplicados para se contrapor aos sintomas característicos do processo de envelhecimento $(23,27,28)$, sendo útil na manutenção da saúde do tecido ósseo $(38,39,40)$ e do volume de massa muscular (41), além de controlar ou reduzir o percentual de gordura corporal (1). $\mathrm{Na}$ maioria dos estudos que procuraram verificar os efeitos do exercício físico sobre o os componentes corporais, especialmente em relação ao tecido ósseo, foram utilizados programas de treinamento com exercícios resistidos. Por outro lado, exercícios de endurance têm se mostrado efectivos na diminuição do percentual de gordura corporal (1). No entanto, poucos estudos têm associado ambas as modalidades de exercícios com o intuito de promover modificações nos componentes corporais de mulheres. Dessa forma, este estudo, teve por objectivo verificar os efeitos de um treinamento constituído por exercícios combinando força e endurance sobre componentes corporais de mulheres na fase de perimenopausa.

\section{METODOLOGIA}

Participaram voluntariamente deste estudo oito mulheres que se encontravam em fase de perimenopausa, seleccionadas através da divulgação em meios de comunicação locais.

As mulheres avaliadas assinaram o termo de consentimento livre e esclarecido, autorizando sua participação na metodologia e concordando com a divulgação dos resultados, sendo guardadas as identidades pessoais. Todos os procedimentos metodológicos aplicados foram aprovados pelo Comité de Ética em Pesquisa com Seres Humanos da Instituição onde este estudo foi desenvolvido.

Os critérios de inclusão assumidos no estudo foram que as envolvidas deveriam ser sedentárias, não apresentarem doenças, e nunca terem participado de programas de exercícios físicos sistematizados, além de não estarem fazendo uso de nenhuma medicação que pudesse interferir nos resultados, estas informações foram obtidas por meio de uma anamnese prévia. Também foram esclarecidos os critérios de exclusão, que seriam a não assiduidade às sessões de treino, assim como o início da utilização de medicamentos que viessem a interferir nos resultados. As características do grupo estudado são apresentadas na Tabela 1.

Tabela 1. Variáveis de caracterização do grupo estudado. Valores expressos em média \pm desvio-padrão ( $n=8$ ].

\begin{tabular}{lcc}
\hline Variáveis & Pré-Treinamento & Pós-Treinamento \\
\hline Massa corporal $(\mathrm{kg})$ & $58,9 \pm 9,3$ & $58,2 \pm 8,1$ \\
Estatura $(\mathrm{cm})$ & $159 \pm 7$ & $159 \pm 7$ \\
Idade $($ anos $)$ & $48,6 \pm 2,1$ & $48,9 \pm 2,2$ \\
VO $_{2}$ máx $\left(\mathrm{ml} \cdot \mathrm{kg}^{-1} \cdot \mathrm{min}^{-1}\right)$ & $27,5 \pm 6,2$ & $30,2 \pm 4,3$ \\
\hline
\end{tabular}

\section{Avaliação dos componentes corporais}

Os componentes corporais avaliados foram a massa óssea (MO), massa gorda (MG) e massa magra (MM). Todas estas variáveis foram determinadas em duas situações (pré e pós-treinamento), a partir de um exame DEXA (Dual Energy X-Ray A bsorptiometry), realizado utilizando-se um equipamento $H$ ologic 
Corporation Qdr 4500 (Waltham, USA). O equipamento foi operado por um profissional com experiência neste tipo de avaliação.

\section{Determinação do consumo máximo de oxigênio ( $\mathrm{VO}_{2}$ máx)}

As alterações no $\mathrm{VO}_{2}$ máx, devido ao programa de treinamento aplicado foram avaliadas a partir dos escores obtidos em testes progressivos máximos (TPM) conduzidos antes e depois do período de treinamento. Para a realização destes testes foi utilizado um analisador de gases VMAX 229 Series (Sensor $M$ edics, Yorba Linda, CA, Estados Unidos) que possibilitou a análise da troca de gases a cada expiração, e uma esteira rolante (Imbramed ATL 10200, Brasil). O protocolo de avaliação utilizado era iniciado com velocidade de $5,4 \mathrm{~km} / \mathrm{h}$, seguidos de acréscimos de $1,8 \mathrm{~km} / \mathrm{h}$ a cada 4 minutos. O término do teste foi determinado quando a exaustão voluntária máxima era atingida, sendo o $\mathrm{VO}_{2}$ máx determinado pelo maior valor observado durante o teste.

\section{Programa de Treinamento}

O período de treinamento teve duração de 20 semanas, onde os sujeitos envolvidos tiveram a frequência mínima de 3 sessões semanais, o não comprimento deste critério consistia na exclusão do grupo de estudo. As sessões combinavam exercícios de força e de endurance. $\mathrm{O}$ treinamento de endurance era realizado no período que precedia o treinamento de força (exercícios resistidos com pesos), sendo constituído de 30 minutos de caminhada, conduzida com intensidade controlada pela velocidade da esteira e relativa a $65 \%$ da velocidade máxima obtida no TPM. Após este período eram conduzidos exercícios de alongamento.

Na sequência, eram iniciados os exercícios resistidos com pesos (2 séries de 20 repetições máximas), sendo a carga aumentada sempre que 20 repetições máximas fossem excedidas. A carga inicial foi baseada na experiência do avaliador na prescrição de exercícios resistidos com pesos, não se utilizou metodologias que consideravam o teste de $1 \mathrm{RM}$, por se tratarem de mulheres sem nenhuma experiência anterior em exercícios resistidos com pesos. Os exercícios utilizados foram abdução e adução de quadril, flexão de joelhos, pressão de pernas, abdominais, supino, flexão de cotovelo, puxada alta, extensão de cotovelo em polia, flexão plantar com joelhos flexionados e elevação lateral, inicialmente um exercício por grupo muscular realizado de modo alternado por segmento, após 4 semanas passou-se a utilizar dois exercícios por grupo muscular, realizados na sequência com um período de recuperação de 1 minuto entre as séries e 1 minuto entre exercícios.

Durante todo o período que consistiu o treinamento nenhuma forma de reposição hormonal, suplementação vitamínica ou qualquer outra forma de controle dietético foi administrada aos sujeitos deste estudo.

\section{Procedimentos estatísticos}

Todos os procedimentos estatísticos foram desenvolvidos no pacote estatístico SPSS 11.5 for Windows. As variáveis de caracterização do grupo de estudo e escores encontrados para MO, MG, MM e percentual de alteração para as situações de pré e pós-treinamento, foram analisados por meio da estatística descritiva, sendo apresentadas em média e desviopadrão. Devido o pequeno grupo amostral, foi utilizado o teste não-paramétrico de Wilcoxon para comparar os valores de pré e pós-teste, sendo assumido um nível de significância de $\mathrm{p}<0,05$.

\section{RESULTADOS}

Inicialmente serão apresentados os percentuais de modificação observados após a intervenção para o $\mathrm{VO}_{2}$ máx. Foram encontrados aumentos tanto para valores relativos $(9,82 \%)$, quanto para valores absolutos $(7,23 \%)$, no entanto esses aumentos não alcançaram significância estatística (Figura 1).

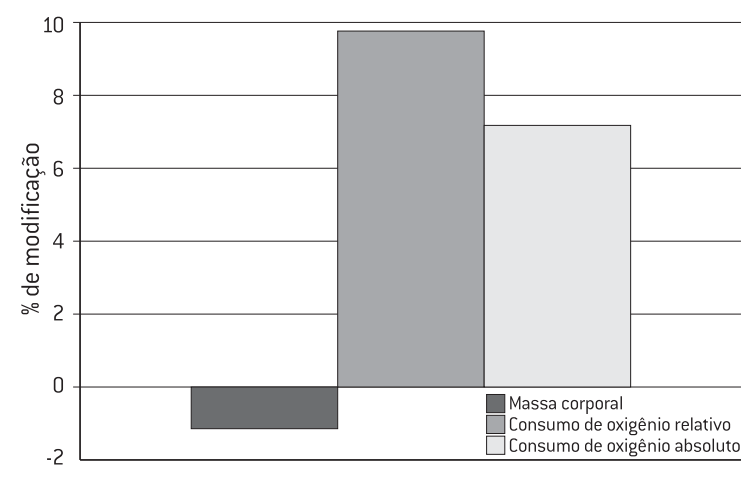

Figura 1. Alterações em relação aos valores de Pré-teste, para a massa corporal, $\mathrm{VO}_{2}$ relativo e $\mathrm{VO}_{2}$ absoluto 
A seguir são apresentados os resultados referentes aos componentes corporais MO, MG e MM, assim como os percentuais de modificação observados após o término programa de exercícios, em relação à massa corporal total. Valores absolutos estão apresentados na Tabela 2, e valores relativos na Tabela 3 . Os percentuais de modificação encontram-se na Figura 2 e Figura 3.

Tabela 2. Valores absolutos individuais, média e desvio-padrão (dp) para massa óssea (MO), massa gorda (MG) e massa magra (MM) no pré e pós-treinamento.

\begin{tabular}{lcccccc}
\hline Sujeitos & \multicolumn{2}{c}{$M 0(\mathrm{~kg})$} & \multicolumn{2}{c}{$M G(\mathrm{Kg})$} & \multicolumn{2}{c}{$M M(\mathrm{~kg})$} \\
& Pré & Pós & Pré & Pós & Pré & Pós \\
\hline 1 & 2,29 & 2,24 & 14,43 & 15,29 & 37,84 & 36,9 \\
2 & 2,07 & 2,07 & 17,84 & 18,08 & 32,41 & 33,64 \\
3 & 2,43 & 2,45 & 23,6 & 20,6 & 43,01 & 43,37 \\
4 & 1,94 & 1,98 & 14,83 & 13,55 & 36,02 & 37,94 \\
5 & 2,15 & 2,18 & 22,99 & 19,82 & 39,58 & 38,23 \\
6 & 1,81 & 1,8 & 16,15 & 16,24 & 29,53 & 30 \\
7 & 1,79 & 1,75 & 14,33 & 14,22 & 33,22 & 34,48 \\
8 & 1,85 & 1,79 & 26,2 & 25,33 & 39,68 & 41,12 \\
\hline Média & 2,04 & 2,03 & 18,8 & 17,89 & 36,41 & 36,96 \\
dp & 0,23 & 0,25 & 4,75 & 3,93 & 4,47 & 4,25 \\
\hline
\end{tabular}

Em relação aos valores absolutos dos componentes corporais, dos sujeitos envolvidos, observou-se que o programa de treinamento não se mostrou efectivo na modificação significativa de nenhum dos componentes corporais analisados, apesar de observa-se uma pequena tendência de modificação em direcção aos propósitos do estudo.

Na Figura 2, estão representadas as alterações percentuais nos componentes corporais analisados.

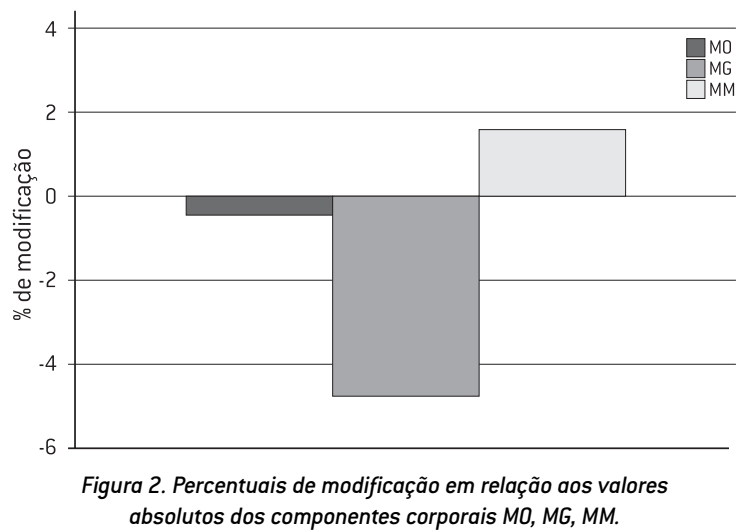

Entre os três componentes analisados o que mais apresentou modificações foi a MG com uma diminuição de $4,83 \%$, seguido da MM com um aumento de $1,51 \%$ e $\mathrm{MO}$ com uma diminuição 0,48. Em relação aos valores dos componentes corporais relativos à massa corporal, os respectivos valores individuais, médias e desvios padrão estão apresentados na Tabela 3.

Tabela 3. Valores relativos individuais, média e desvio-padrão (dp) para massa óssea (MO), massa gorda (MG) e massa magra (MM) no pré e pós-treinamento.

\begin{tabular}{lcccccc}
\hline Sujeitos & \multicolumn{2}{c}{ MO $(\%)$} & \multicolumn{2}{c}{$M G(\%)$} & \multicolumn{2}{c}{$M M(\%)$} \\
& Pré & Pós & Pré & Pós & Pré & Pós \\
\hline 1 & 4,1 & 3,99 & 25,77 & 27,3 & 67,57 & 65,9 \\
2 & 3,83 & 3,77 & 33,04 & 32,87 & 60,02 & 61,17 \\
3 & 3,37 & 3,57 & 32,78 & 30,08 & 59,74 & 63,31 \\
4 & 3,59 & 3,6 & 27,46 & 24,63 & 66,7 & 68,99 \\
5 & 3,2 & 3,57 & 34,31 & 32,49 & 59,07 & 62,68 \\
6 & 3,72 & 3,67 & 33,3 & 33,13 & 60,89 & 61,23 \\
7 & 3,59 & 3,51 & 28,66 & 28,44 & 66,44 & 68,95 \\
8 & 2,65 & 2,52 & 37,43 & 35,68 & 56,68 & 57,92 \\
\hline Média & 3,46 & 3,49 & 31,89 & 30,75 & 61,78 & 63,52 \\
dp & 0,44 & 0,43 & 3,92 & 3,63 & 4,14 & 3,92 \\
\hline
\end{tabular}

Assim como fora observado nos valores absolutos, também nos valores relativos a massa corporal, não foram encontradas diferenças estatisticamente significativas entre as situações pré e pós-treinamento. Os percentuais de alteração dos componentes corporais relativos a massa corporal estão apresentados na Figura 3.

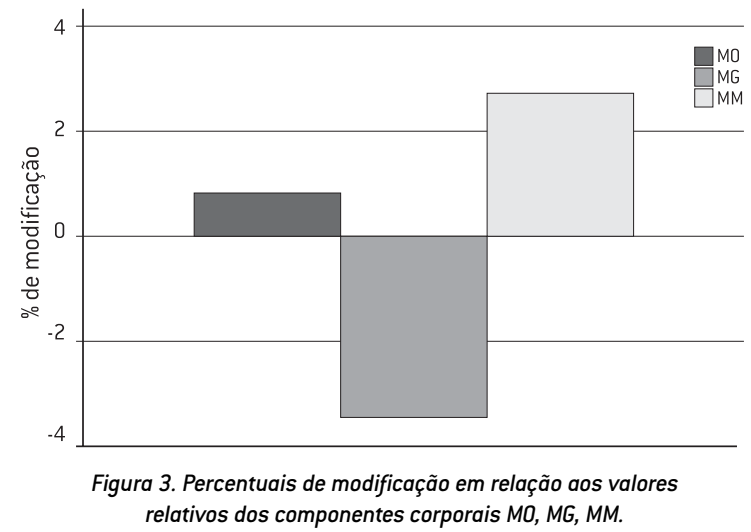


Em relação às modificações percentuais observadas para os componentes corporais apresentados na Figura 3 , para nenhum destes houve modificações estatisticamente significativas, sendo o componente que mais representou esta característica foi a MG, com uma diminuição de $3,6 \%$, seguido da MM com um aumento de $2,82 \%$ e da $\mathrm{MO}$ com um aumento de $0,81 \%$.

\section{DISCUSSÃO}

Com base na literatura revisada, com o processo de envelhecimento ocorrem alterações como a diminuição do percentual de massa óssea, aumento do percentual de massa gorda e diminuição do percentual de massa muscular $(7,32,5,20,42)$. O objectivo deste estudo foi justamente verificar se um período de treinamento poderia afectar esse processo natural. Apesar de nenhuma das variáveis terem apresentado alterações estatisticamente significativas, o que poderia representar a ineficiência do programa de treinamento, cabe ressaltar que o programa de exercícios foi responsável pela estagnação das características naturais desta fase da vida em relação aos componentes corporais, além de demonstrarem uma tendência no sentido oposto ao processo natural do envelhecimento, no entanto, isso poderia ser evidenciado através da utilização de um grupo controle que não foi possível neste estudo.

A partir da observação dos resultados obtidos para o grupo de estudo em relação ao percentual de gordura, podemos classificar os sujeitos como acima da média esperada em relação à saúde, segundo parâmetros estabelecidos por Lohman (24). Apesar de ter sido encontrado uma diminuição de $3,6 \%$ no percentual de gordura em relação ao pré-treinamento, os sujeitos ainda se encontram em níveis considerados elevados e propícios para o desenvolvimento de algumas doenças de cunho metabólico (20).

O Colégio Americano de Medicina do Esporte (1) recomenda que para a redução da MG, estejam associados exercícios regulares e controle de dieta. Como neste estudo não foram controlados aspectos relacionados à dieta dos sujeitos, uma possível explicação encontrada para a não diminuição significativa dos valores de MG poderia ser as características do treinamento aeróbico utilizado neste estudo (30 minutos, $65 \%$ da velocidade máxima no TPM-Pré), ou seja o programa de treinamento aeróbio não foi suficientemente intenso para que alterações significativas fossem obtidas .
A modificação observada neste estudo para a MG foram inferiores ao apresentado por Stewart et al. (35), que apresentou uma redução de 3,36 kg após 6 meses de treinamento. No entanto, estes autores fizeram um controle rígido da dieta, além de um período superior de actividades aeróbicas ( $45 \mathrm{~min}$ ) com intensidades também superiores ao utilizado no presente estudo (60 a $90 \%$ da FCmáx).

Em relação a MM, observa-se sua diminuição como um dos factores relacionados ao envelhecimento, sendo ainda associada com consequentemente diminuição da força muscular (37), que é um dos principais factores responsáveis pela maioria das quedas e fracturas ocorridas em idosos (10). O treinamento de força tem se mostrado efectivo como ferramenta no combate a perda de conteúdo proteico muscular, além de promover aumentos neste componente corporal tanto para mulheres como homens, mesmo em idades mais avançadas $(8,40)$.

Em relação a MM, observou-se uma modificação de $2,82 \%$, o que corresponde a pouco mais de $0,5 \mathrm{~kg}$. Esta alteração é menor do que o apresentado por Stewart et al. (35) que relataram aumento de $1,17 \mathrm{~kg}$ na MM de mulheres após um programa de treinamento. No entanto, assim como fora citado anteriormente as características de volume, intensidade e tempo em que o estudo se deu, foram superiores ao desenvolvido neste estudo.

A maioria dos estudos revisados que buscaram avaliar os aspectos do tecido ósseo em relação ao processo de envelhecimento preocupou-se basicamente com as alterações na densidade mineral óssea, uma vez que esta tem maior capacidade de discriminação em relação às modificações em locais específicos do corpo, como por exemplo, na coluna lombar e cabeça do fémur. Isto se dá em função de estes serem os locais mais acometidos por fracturas e problemas osteo-articulares com o avançar da idade. Devido a isto, poucos estudos se dedicam a discutir valores absolutos e relativos de MO, o que dificultou o encontro de parâmetros a fim de comparação para esta variável.

Dos componentes corporais analisados, o que menor mudança apresentou foi a MO. As explicações encontradas na literatura que sustentariam tal comportamento, poderiam estar ligadas ao fato dos sujeitos envolvidos no estudo estarem na fase de perimenopausa, a qual é caracterizada pela aceleração da perda de massa óssea ${ }^{(36,14)}$, o que os tornaria 
menos propensos a apresentarem respostas positivas ao treinamento. Associado a isto também está o fato de que o tecido ósseo apresenta uma resposta mais lenta a cargas de trabalho quando comparado a outros sistemas, como, por exemplo, o muscular e o cardiovascular (4).

O tempo necessário para que as estruturas ósseas respondam a um programa de treinamento ainda não está bem claro na literatura, mas estudos com duração semelhante ao deste trabalho $(12,16)$, também fracassaram na perspectiva de encontrar melhorias nos componentes ósseos após programas de exercícios.

Isto foi observado no estudo desenvolvido por Humphries et al. (16) onde um período de 24 semanas não encontrou melhoras na DMO da coluna lombar de mulheres. O diferencial deste estudo foi a utilização de quatro grupos, divididos em duas formas de exercício (caminhada e exercícios com pesos livres), sendo que dois grupos ainda fizeram uso de estratégias de reposição hormonal. Os autores encontraram, para todos os grupos avaliados, uma diminuição na DMO, sendo esta somente significativa estatisticamente para o grupo de caminhada. Ainda neste contexto, outro estudo que fracassou ao tentar mostrar melhoria da DMO foi o conduzido por Hartard et al. (12) que após conduzir um programa de treinamento com pesos por um período de seis meses (intensidade de $70 \%$ de 1RM) não encontrou diferenças significativas na DMO da coluna lombar (L2-L4), tampouco no fémur. No entanto, para o grupo controle, no mesmo período observou-se uma diminuição de $6,2 \%$ na DMO da coluna lombar.

Assim, o que foi encontrado parece condizer com achados de outros estudos, onde os programas de exercício com duração de até 6 meses teriam uma função de manutenção da massa óssea e não propriamente aumentar seu conteúdo, no entanto existiria a necessidade de possuir um grupo controle, o que agregaria valor à resposta da MO após as 20 semanas de treinamento.

O início da fase de menopausa tem como consequência a redução dos valores de consumo máximo de oxigénio (26), no entanto, o emprego de programas de exercícios físicos tem se mostrado ferramentas efetivas na promoção de aumentos em seus valores máximos (18). Os dados deste estudo apontaram para um aumento, ainda que não significativo, tanto nos valores de $\mathrm{VO}_{2}$ máx relativos $(9,82 \%)$, quanto para valores de $\mathrm{VO}_{2}$ máx absolutos $(7,23 \%)$, sendo que as possíveis causas de tais aumentos seriam as alterações de $2,82 \%$ na MM, 3,6\% na MG. Isso seria devido ao fato que a massa muscular, componente da MM é a principal variável responsável pelas variabilidades no $\mathrm{VO}_{2}$ máx, tanto para homens quanto para mulheres, com idade inferior a 60 anos $(8,9)$. As diferenças encontradas neste estudo foram inferiores aos encontrados Kemmler et al. (18), no entanto, as diferenças de $13,9 \%$ de aumento nos valores de $\mathrm{VO}_{2}$ máx encontrados pelos autores foram obtidos após 3 anos de treinamentos, tempo bem superior ao desenvolvido neste estudo.

\section{CONCLUSÃO}

De acordo com os resultados obtidos neste estudo, pode-se concluir que um programa de exercícios físicos combinando força e endurance durante um período de 20 semanas não foi suficiente para promover alterações significativas tanto nos componentes corporais estudados, quanto no $\mathrm{VO}_{2}$ máx de mulheres na fase de perimenopausa, seja ele absoluto ou relativo. No entanto, observou-se uma tendência de manutenção ou modificações em todos os componentes corporais avaliados, sugerindo que um tempo superior ao utilizado nesta investigação possa ser mais eficiente.

Com isso sugere-se a realização de trabalhos com um grupo de estudo maior além de utilizar-se um grupo como controle, onde a intensidade de exercício e o período de treinamento sejam superiores aos aplicados neste estudo, também se sugere o controle de variáveis intervenientes que neste estudo não foram controladas, mas que são sabidamente responsáveis pela modificação dos componentes corporais, como a reposição hormonal, suplementação vitamínica e os aspectos nutricionais.

\section{CORRESPONDÊNCIA \\ Mateus Rossato}

Universidade Federal de Santa Catarina

Centro de Desportos, Laboratório de Biomecânica

Campus Universitário Trindade

CEP: 88040-900 Florianópolis - SC - Brasil

Telefone: 55483331 8530; Fax: 04833319927

E-mail: mateusmestrado@gmail.com 


\section{REFERÊNCIAS}

1. ACSM's. (2006) Guidelines for exercise testing and prescription, $7^{\circ}$ Edição, Rio de Janeiro: Guanabara Koogan S.A.

2. Brozek J, Kihlberg JK, Taylor L, Keys A (1963). Skinfolds distributions in middle-aged american men: a contribution to norms of leanness-fatness. A nnals N ew York A cademy Science 110: 492-502.

3. Cheng S, Sipila S, Taaffe DR, Puolakka J, Suominen H. (2002). Change in bone mass distribution induced by hormone replacement therapy and high-impact physical exercise in post-menopausal women. Bone 31(1):126-135

4. Cullen DM, Iwaniec UT, Bargar-Lux MJ (2003). Resposta esquelética no exercício e no treinamento. In: A ciência do exercício e dos esportes. Garrett Jr. WE. \& Kirkendall DT. e cols. Artmed, 251-262.

5. Douchi T, Yamamoto S, Oki T, Maruta K, Kuwahata R, Yamasaki H, Nagata Y(2000). Difference in the effect of adiposity on bone density between pre- and postmenopausal women. M aturitas 34: 261-266

6. Drinkwater BL.(1993). Exercise in the prevention of osteoporosis. In: Osteoporosis, Proceedings, C. Christiansen and B. Riis (Eds.). Rodovre, Denmark Osteopress Aps., 105-108.

7. Durnin JVG. (1983). A body composition and energy expenditure in elderly people. Bibliothca N utr. Dieta 33: 16 30 .

8. Evans WJ. (2003). Envelhecimento e exercício. In: A ciência do exercício e dos esportes. Garrett Jr. WE. \& Kirkendall DT. e cols. Artmed: 302-309.

9. Fleg JL.; Lakatta EG. (1988). Role of muscle loss in the age-associated reduction in $\mathrm{VO}_{2}$ máx. J A ppl. Physiol 65 1147-1151.

10. Frontera WR, Hughes VA, Evans WJ. (1991). A cross-sectional estudy of upper and lower extremy muscle strength in 45-78 years old men and woman. J A ppl Physiology 71: 644-650.

11. Gleeson PB, Protas EJ, Leblanc AD, Schneider VS, Evans HJ (1990). Effects of weight training on bone mineral density in premenopausal women. J Bone M iner Res 5: 53-158.

12. Hartard M, Haber P, Ilieva D, Preisinger E, Seidl G, Huber J (1996). Systematic strength training as a model of therapeutic intervention: a controlled trial in postmenopausal women with osteoporosis. A m J Phys M ed Rehab 75: 21-28.

13. Hewitt MJ, Williams DP, Going SB, Lohman TG (1991). Skinfold Estimation of percent fat from measures of density, water and bone in middle-aged and older men and women. M ed Sci Sports Exerc 23(4): 149.

14. Hill C. (2001). Caring for the aging athlete. Geriatric Nursing 22 (1): 43-45.

15. Hoover PA, Webber CE, Beaumont LF, Blake JM. (1996). Postmenopausal bone mineral density: relationship to calcium intake, calcium absorption, residual estrogen, body composition, and physical activity. Can J Physiol Pharmacol 74: 911-917.

16. Humphries B, Newton RU, Bronks R, Marshall S, Mcbride J, Triplett-Mcbride T, Hakkinen K, Kraemer WJ, Humphries N (2000). Effect of exercise intensity on bone density, strength, and calcium turnover in older women. M ed Sci Sports Exerc 32(6): 1043-1050.

17. Ilich JZ, Kerstetter JE (2000). Nutrition in bone health revisited: A story beyond calcium. Journal of the A merican College of Nutrition 19(6), 715-737.
18. Kemmler W, Von Stengel S, Weineck J, Lauber D, Kalender W, Engelke K (2005). Exercise Effects on Menopausal Risk Factors of Early Postmenopausal Women: 3-yr EFOPS Results. M ed Sci Sports Exerc 37(2): 194-203.

19. Khandwala SS (1998). Primary care of the perimenopausal woman. Primary Care U pdate for OB/GYNS 5(1): 43-49(7)

20. Kontogianni MD, Panagiotakos DB, Skopouli FN (2005). Does body mass index reflect adequately the body fat content in perimenopausal women? M aturitas 51: 307-313.

21. Lauritzen JB (1996). Hip fractures: incidence, risk factors, energy absorption, and prevention. Bone 18 (1 Suppl): 65S-75S.

22. Li S, Wagner R, Holm K, Lehotsky J, Zinaman MJ (2004). Relationship between soft tissue body composition and bone mass in perimenopausal women. M aturitas 47: 99-105

23. Lohmam T. (1992). A dvances in body composition assessment. Champaing, IL: Human Kinetics.

24. Lohman T, Going S, Pamenter R, Hall M, Boyden T, Houtkooper L, Ritenbaugh C, Bare L, Hill A, Aickin M (1995). Effects of resistance training on regional and total BMD in premenopausal women: a randomized prospective study. J Bone M iner Res 10(7): 1015-24.

25. Matsudo SM, Matsudo VKR, Barros Neto TL. (2000). Impacto do envelhecimento nas variáveis antropométricas, neuromotoras e metabólicas da aptidão física. Rev. Bras.Ciên. e M ov. 8(4): 21-32.

26. Mercuro G, Saiu F, Deidda M, Mercuro S, Vitale C, Rosano G (2006). Impairment of physical exercise capacity in healthy postmenopausal women. A m H eart J 151(4): 923-927.

27. Nichols JF, Nelson KP, Peterson KK, Sartoris DJ (1995). Responses to high intensity strength training in active older women. J A ging Phys Act 3:26-38.

28. Niemam D. (1999) Exercício e Saúde. São Paulo: Manole.

29. Oliveira R, Pereira MH, Matsudo VKR. (1988) Terceira idade: características antropométricas e consumo de oxigênio em mulheres praticantes e não praticantes de atividade física. Revista Brasileira de Ciência e M ovimento 2(4): 17-21.

30. Posicionamento Oficial do Colégio Americano de Medicina do Esporte Sobre Osteoporose e Exercício (1995). M ed Sci Sports Exerc 27(4).

31. Rockwell JC, Sorensen AM, Baker S, Leahey D, Michaels J. (1990) Weight training decreases vertebral bone density in premenopausal women: a prospective study. J Clin Endocrinol M etab 71:988-993.

32. Shephard RJ. (1994)Alterações fisiológicas através dos anos. In: A merican College Of Sports M edicine. Prova de Esforço e Prescrição de Exercício. Rio de Janeiro: Revinter.

33. Shephard, R. J. (1995). Physical activity, health, and wellbeing at different life stages. R esearch Quarterly for Exercise and Sport 66(4), 298-302.

34. Spirduso, W. W. (2005). Dimensões físicas do envelhecimento. Barueri, São Paulo: Manole.

35. Stewart KJ, Bacher AC, Hees PS, Tayback M, Ouyang P, Jan de Beur S. (2005). Exercise effects on bone mineral density relationships to changes in fitness and fatness. A merican Journal of Preventive M edicine 28 (5): 453-60.

36. Svendsen OL, Hassager C, Christiansen C. (1995). Age- and menopause-associated variations in body composition and fat distribution in healthy women as measured by dual-energy X-ray absorptiometry. M etabolism 44(3): 369-73. 
37. Tanko LB, Movsesyan L, Mouritzen U, Christiansen C, Svendsen OL. (2002). Appendicular lean tissue mass and the prevalence of sarcopenia among healthy women. Metabolism 51(1): 69-74.

38. Turner CH, Robling AG (2003). Designing exercise regimens to increase bone strength. Exerc Sport Sci Rev 31: 45-50.

39. Wallace BA, Cumming RG (2000). Systematic review of randomized trials of the effect of exercise on bone mass in pre- and postmenopausal women. Calcif Tissue Int 67(1):10-8.

40. Wark JD (1996). Osteoporotic fractures: background and prevention strategies. M aturitas 23: 193-207.

41. Weineck J (1999). Treinamento ideal. $9^{\circ}$ edição. São Paulo: Monole.

42. Womersley J, Durnin JV, Boddy K, Mahaffy M (1976). Influence of muscular development, obesity and age on fat-free mass of adults. Journal of A pplied Physiology 41(2): 223- 229. 